\section{Misdiagnosed acute aortic dissection type A}

$\operatorname{JANA} \operatorname{MAKUC}(\bowtie) \cdot$ MARTIN TRETJAK

Slovenj Gradec General Hospital Department of Internal Medicine Gosposvetska 1.

SI - 2380 Slovenj Gradec

Phone: +38628823400

Fax: +386288234505

E-mail: jana.makuc@sb-sg.si

\begin{abstract}
In the absence of diagnostic imaging, chest pain can represent a diagnostic dilemma, especially, if the patient's condition requires immediate action. We present a case of a patient with signs of obstructive shock that turned out to result from an acute aortic dissection (AAD) and consequent cardiac tamponade. Before the correct diagnosis was made, myocardial infarction and/or pulmonary embolism were suspected and the patient was treated with antithrombotic and anticoagulant drugs, which are contraindicated in AAD. We discuss the correct procedures and treatment in suspected AAD.
\end{abstract}

Key words: acute aortic dissection, Stanford type A, misdiagnosis

\section{Introduction}

Acute aortic dissection (AAD) is a medical emergency with a high mortality rate, rising by approx. $1 \%$ per hour. It usually presents with severe, unrelenting chest pain of sudden onset. Pain can be described as ripping or tearing in nature, or stabbing or sharp in character. It can migrate as the dissection extends down the aorta (17\%). Less common signs and symptoms are related to organ hypoperfusion and include peripheral ischemic syndromes (19\%), syncope (13\%), myocardial infarction (13\%), heart failure (8.8\%) and neurologic symptoms (6.1\%). (1-3) Cases of painless AAD have also been described. $(4,5)$ Because of such heterogeneous clinical presentations, many incorrectly diagnosed cases escape notice. Approx. one third of patients ultimately diagnosed with $A A D$ are first thought to have another diagnosis. The true incidence of $A A D$ is therefore difficult to determine, but is estimated to be 5-20/1,000,000. (6)

$A A D$ is classified based on the location and extent of dissection. The two most commonly used classification systems are the DeBakey system, which categorizes aortic dissection based on where the original intimal tear is located and the extent of the dissection (type I - III), and the functional Stanford system, dividing dissections into those involving the ascending aorta (type A, more common) and those distal to the outlet of the left subclavian artery (type B). In general, type A dissections have a higher in-hospital mortality rate (30\%) and require immediate surgical treatment, whereas type $\mathrm{B}$ dissections have a better prognosis (10\% in-hospital mortality) and may be managed medically. The clinical outcome of AAD is eventually determined by dissection type and timing of presentation, patient-related factors and the quality and experience of the individuals and institution providing care. (1)

\section{Case report}

A 52-year old male, previously healthy, was brought to our hospital because of sudden severe chest pain, followed by loss of consciousness few minutes later. The patient's wife had witnessed the event and immediately called the rescue team. She told them the patient was feeling bad the whole day and had vomited a couple of times. On their arrival (within ten minutes), the patient showed signs of obstructive shock: he was soporous, pale and sweaty, had cyanotic lips and evidently full neck veins. During initial assessment his heart rate was normocardic, but was continuously rising during treatment (from 90 to 112 bpm); his blood pressure stayed low (RR 60/35 $\mathrm{mm} \mathrm{Hg}$ ). Besides intravenous fluids, he immediately received oxygen via an Ohio oxygen mask, resulting in a rise in peripheral blood saturation from $82 \%$ to $91 \%$. Because of suspected acute myocardial infarction (AMI) acetylsalicylic acid (500 mg) was administered to the patient. This diagnosis seemed less probable later, when a normal ECG was recorded. Lacking diagnostic imaging, the physician in charge was left wavering between a diagnosis of acute aortic dissection and massive pulmonary embolism and decided for the latter. The patient therefore received 5000 IU of heparin intravenously and was rushed to our facility (a regional 
hospital). There, the patient's clinical condition was unchanged, except for a pulse deficit on the left side of his body. Heart sounds were quiet, but there was no murmur. While waiting for computed tomography angiography (CTA), fast transthoracic echocardiography (TTE) examination was performed, showing a massive inhomogeneous pericardial effusion, most probably partially coagulated blood. CTA confirmed the high suspicion of a haemopericardium, resulting from rupture of a type A aortic dissection. Dissection extended throughout the ascending aorta, also comprising the aortic arch and descending aorta above the branching of the left renal artery. Because the patient's blood pressure and heart rate seemed stable, no additional drugs were given (incl. protamine sulphate) and pericardiocentesis was not performed. The patient was urgently transferred to a tertiary medical centre for operative treatment. There were no changes in his clinical condition during transport, but while being placed on the operating table, he went into cardiac arrest. After successful resuscitation a Bentall operation was performed, but had to be revised because of profuse postoperative haemorrhaging. Two weeks later the patient was discharged from hospital, subjectively feeling very well (except for a mild left arm paresis).

\section{Discussion}

Studies show that $31-39 \%$ of AAD's are initially misdiagnosed, most commonly as AMI, which is said to be "a catastrophe in disguise" because of inappropriate treatment. Exposure to antithrombotic agents in misdiagnosed AAD is associated with significantly higher rates of major bleeding, hemodynamic instability and greater in-hospital mortality. (7-10) Therefore, if $A A D$ is highly suspected drugs influencing blood coagulation should not be administered until diagnosis is excluded. Nevertheless, if an anticoagulant is given anyway, unfractionated heparin (in comparison to low molecular weight heparins) has the advantage of quick and complete reversal by protamine sulphate.

While waiting for an appropriate imaging method (computed tomography angiography - CTA, magnetic resonance imaging (MRI), transoesophageal echocardiography (TEE) to confirm or exclude the diagnosis, it is necessary to control pain and reduce systolic blood pressure to values between 100 and $120 \mathrm{mmHg}$. Patients with profound hemodynamic instability need to be intubated and ventilated without delay. It remains controversial whether pericardiocentesis should be performed in the case of pericardial tamponade; this may be harmful as it lowers pericardial pressure and can cause recurrent bleeding. When urgent surgery is needed, TEE in combination with TTE can be used as the sole diagnostic procedure. If more spatial resolution is necessary, CTA or MRI is used in addition. Some authors also recommend coronary angiography for all stable patients, because chronic coronary atherosclerotic disease (present in 25\% of patients) may worsen the surgical outcome, but this remains undetermined. (11)

The aims of surgical intervention in type A (type I and II) AAD are to prevent aortic rupture and pericardial effusion leading to cardiac tamponade, to relieve aortic regurgitation and to prevent myocardial ischemia. A large variability of surgical techniques exists, depending on the anatomic conditions and the condition of the aortic valve. (11)

Although the clinical outcome is determined by a variety of factors, the key in management of $A A D$ is to maintain a high level of suspicion for this diagnosis.

\section{REFERENCES}

1. Weigang E, Nienaber CA, Rehders TC, Ince H, Vahl CF, Beyersdorf F. Management of patients with aortic dissection. Dtsch Arztebl Int 2008;105(38):639-46.

2. Noč M. Sudden excruciating chest pain - prehospital management. In: Grmec Š, Tušek-Bunc K, Kupnik D, editors. Acute conditions: signs, symptoms, syndromes, differential diagnosis and management: proceedings and algorithms. Maribor: Slovene Family Medicine Society SZD; 2003. p. 21-4.

3. Benko D, Tretjak M, Rainer S. Cardiac tamponade resulting from acute aortic dissection type A - case report and diagnostic approach. In: Bručan A, Gričar M, Klančar S, Fink A, editors. Emergency medicine: selected topics 6. Ljubljana: Slovenian Society for Emergency Medicine; 2000. p. 441-6.

4. Ayrik C, Cece H, Aslan O, Karcioglu O, Yilmaz E. Seeing the invisible: painless aortic dissection in the emergency setting. Emerg Med J 2006;23(3):24.

5. Nadour W, Goldwasser B, Biederman RW, Taffe K. Silent aortic dissection presenting as transient locked-in syndrome. Tex Heart Inst J 2008; 35(3):359-61.

6. Auer J, Berent R, Eber B. Aortic dissection: incidence, natural history and impact of surgery. J Clin Basic Cardiol 2000;3:151-4. 
7. Hansen MS, Nogareda GJ, Hutchison SJ. Frequency of and inappropriate treatment of misdiagnosis of acute aortic dissection. Am J Cardiol 2007;99(6):852-6.

8. Luo JL, Wu CK, Lin YH, Kao HL, Lin MS, Ho YL, et al. Type A aortic dissection manifesting as acute myocardial infarction: still a lesson to learn. Acta Cardiol 2009;64 (4):499-504.

9. Asouhidou I, Asteri T. Acute aortic dissection: be aware of misdiagnosis. BMC Res Notes 2009;2:25.

10. Marek D, Nemec P, Herman M, Gwozdziewicz M, Troubil M, Lukl J. Mistakes in dealing with aortic dissection. Lessons from three warning cases. Biomed Pap Med Fac Univ Palacky Olomouc Czech Repub 2008;152(2):283-27.

11. Erbel R, Alfonso F, Boileau C, Dirsch O, Eber B, Haverich A, et al. Recommendations of the Task Force on Aortic Dissection, European Society of Cardiology. Diagnosis and management of aortic dissection. Eur Heart J 2001;22:1642-81. 